International Journal of Biomedicine I June 2019 - Volume 9, Issue Suppl_1: Abstracts From the Second Russian International Conference "Cryo-electron microscopy 2019: achievements and prospects"

POSTER ABSTRACT PRESENTATIONS

SESSION TITLE: STRUCTURE OF VIRUSES AND CHAPERONINS

DOI: 10.21103/IJBM.9.Suppl_1.P28

\title{
Abstract P-28: Protocol of Tick-Borne Encephalitis Virus Sample Preparation for Structural Studies
}

\author{
Anton Y. Fedotov ${ }^{1}$, Mikhail F. Vorovitch ${ }^{1,3}$, Kseniya K. Tuchinskaya ${ }^{1}$, Konstantin V. Grishin ${ }^{1}$, \\ Olga I. Konyushko ${ }^{1}$, Dmitry I. Osolodkin ${ }^{1,2}$, Aidar A. Ishmukhametov ${ }^{1}$, Alexey M. Egorov ${ }^{1,2}$ \\ ${ }^{1}$ FSBSI “Chumakov FSC R\&D IBP RAS”, Moscow, Russia; ${ }^{2}$ Lomonosov Moscow State University, \\ Moscow, Russia; ${ }^{3}$ Sechenov First Moscow State Medical University, Moscow, Russia
}

Background: Tick-borne encephalitis virus (TBEV), a member of genus Flavivirus, is an important human pathogen: up to 10,000 cases of TBE disease are registered in Europe and Asia annually. Inactivated vaccines are widely used to prevent the disease. The structure of viral particles forming the vaccine antigen was never studied in details sufficient to characterize them on the atomic level. Thorough characterization of the inactivated viral particles would allow their comparison with the native virus particles for the rational structure-based vaccine design. Such information is important for understanding the virus neutralization and inactivation mechanisms, as well as for the comparison of the epitopes inducing the immonological response in the inactivated preparations and wild-type virions.

X-Ray Free Electron Lasers (XFEL) provide a unique opportunity to study the time-resolved solution structures of large macromolecular assemblies, such as viruses. In the single particle approach, a suspension of the viral particles is injected into the X-ray beam with the help of electrospray. The method requires substantially high concentration of particles, homogeneity, absence of aggregates and large volume.

Methods: The prototype TBEV strain Sofjin was chosen as the model for developing the flavivirus sample preparation protocol for XFEL studies. Viral particles in the resulting samples were quantified by ELISA and RT-PCR. Negative staining electron microscopy and cryoelectron miscroscopy are the methods of choice for the quality control of flavivirus preparation. As flavivirus virions are enveloped, their preparation for electrospray injection requires a characterization of the particle rigidity.

Results: TBEV was grown using primary or continuous cell cultures. Several protocols, based on filtration, centrifugation, and chromatography, were applied to purify inactivated TBEV particles. The concentration of the sample obtained according to the optimized protocol was $\sim 10^{13} \mathrm{~mL}^{-1}$. 
Conclusion: The purification protocol based on the combination of tangential flow filtration and two steps of ultracentrifugation, one of them through the sucrose gradient, was the most efficient for obtaining high concentrated, non-aggregated virus suspension suitable for XFEL studies.

Key Words: TBEV • structure • XFEL

Sources of Funding: This work is supported by the Russian Foundation for Basic Research (grant no. 1802-40026).

International Journal of Biomedicine. 2019;9 Suppl 1: S29. doi: 10.21103/IJBM.9.SuppI_1.P28

(C)2019 International Medical Research and Development Corporation 